\title{
Extension of Optimal Homotopy Asymptotic Method with Use of Daftardar-Jeffery Polynomials to Coupled Nonlinear-Korteweg- De-Vries System
}

\author{
Rashid Nawaz $\mathbb{D}^{1},{ }^{1}$ Zawar Hussain, ${ }^{1}$ Abraiz Khattak, ${ }^{2}$ and Adam Khan $\mathbb{D D}^{3,4}$ \\ ${ }^{1}$ Department of Mathematics, Abdul Wali Khan University, Mardan, KP, Pakistan \\ ${ }^{2}$ Department of Electrical Power Engineering, U.S.-Pakistan Center for Advanced Studies in Energy (USPCAS-E), \\ National University of Sciences and Technology, Islamabad (44000), Pakistan \\ ${ }^{3}$ Department for Management of Science and Technology Development, Ton Duc Thang University, Ho Chi Minh City, Vietnam \\ ${ }^{4}$ Faculty of Electrical \& Electronics Engineering, Ton Duc Thang University, Ho Chi Minh City, Vietnam \\ Correspondence should be addressed to Adam Khan; adamkhan@tdtu.edu.vn
}

Received 25 July 2019; Revised 3 November 2019; Accepted 28 November 2019; Published 9 March 2020

Guest Editor: Raúl Baños

Copyright (c) 2020 Rashid Nawaz et al. This is an open access article distributed under the Creative Commons Attribution License, which permits unrestricted use, distribution, and reproduction in any medium, provided the original work is properly cited.

In this paper, Daftardar-Jeffery Polynomials are introduced in the Optimal Homotopy Asymptotic Method for solution of a coupled system of nonlinear partial differential equations. The coupled nonlinear KdV system is taken as test example. The results obtained by the proposed method are compared with the multistage Optimal Homotopy Asymptotic Method. The results show the efficiency and consistency of the proposed method over the Optimal Homotopy Asymptotic Method. In addition, accuracy of the proposed method can be improved by taking higher order approximations.

\section{Introduction}

Differential equations play a vital role in engineering and applied sciences. The nonlinear coupled Partial Differential Equations (PDEs) have a variety of applications in physics, acoustics, optics, elasticity, hydrodynamics, aerodynamics, electromagnetism, chemical kinetics, economics, computer science, and financial mathematics. The exact solutions of nonlinear PDEs cannot be found easily. Different approaches have been adopted by researchers for the approximate solutions of these equations. The well-known approaches are Runge-Kutta method (R-K) [1, 2], Shooting method (SM) [3, 4], Finite Difference Method (FDM) [5-7], Finite Element Method (FEM) $[8,9]$, Collocation Method [10-13], and Homotopy Analysis Method (HAM) [14, 15]. Recently, Marinca et al. introduced the Optimal Homotopy Asymptotic Method (OHAM) for the solution of nonlinear problems [16-18] which made the perturbation methods independent of the hypothesis of small parameters and huge computational work. They used more flexible function called the auxiliary function which controls the convergence of the proposed method. To improve the accuracy and to ensure the faster convergence, one can use an increased number of convergence control parameters in the first order of approximation. A more general formulation, which emphasizes the above features, was presented in $[19,20]$. Later on, Ali et al. introduced a new method based on adaptation of the Optimal Homotopy Asymptotic Method (OHAM) with Daftardar-Jeffery polynomials, called OHAM-DJ, to solve nonlinear problems $[21,22]$. Shah et al. applied OHAM-DJ for the solution of linear and nonlinear Klein-Gordon equations [23]. Our main goal in this work is to extend the applications of OHAM-DJ to a coupled nonlinear KdV system. 

form:

The coupled nonlinear $\mathrm{KdV}$ system has the following

$$
\begin{gathered}
\frac{\partial \zeta(\eta, t)}{\partial t}-0.5 \frac{\partial^{3} \zeta(\eta, t)}{\partial \eta^{3}}+3 \zeta(\eta, t) \frac{\partial \zeta(\eta, t)}{\partial \eta} \\
-3 \frac{\partial}{\partial \eta} \zeta(\eta, t) w(\eta, t)=0 \\
\frac{\partial \xi(\eta, t)}{\partial t}+\frac{\partial^{3} \xi(\eta, t)}{\partial \eta^{3}}-3 \zeta(\eta, t) \frac{\partial \xi(\eta, t)}{\partial \eta}=0 \\
\frac{\partial w(\eta, t)}{\partial t}+\frac{\partial^{3} w(\eta, t)}{\partial \eta^{3}}-3 \zeta(\eta, t) \frac{\partial w(\eta, t)}{\partial t}=0
\end{gathered}
$$

\section{Basic Idea of OHAM-DJ}

Consider the nonlinear differential equation:

$$
L(\zeta(\eta, t))+N(\zeta(\eta, t))+g(\eta, t)=0, \quad \eta \in \Omega, B\left(\zeta, \frac{\partial \zeta}{\partial t}\right)=0
$$

In equation (2), $L$ is a linear operator, $N$ is a nonlinear operator, $\zeta$ is an unknown function, $g$ is a known function, and $B$ is a boundary operator. According to OHAM-DJ, the optimal homotopy $H(\varphi(\eta, t ; s)): \Omega \times[0,1] \longrightarrow R$ satisfies the following equation:

$$
\begin{aligned}
(1-s)[L(\varphi(\eta, t ; s))+g(\eta, t)]= & H(s)[L(\varphi(\eta, t ; s)) \\
& +N(\varphi(\eta, t ; s))+g(\eta, t)] .
\end{aligned}
$$

In equation (3), $s \in[0,1]$ is an embedding parameter, $H(s)$ is a nonzero auxiliary function for $s \neq 0$, and its value is zero for $s=0$. The unknown function $\zeta(\eta, t ; s)$ starts from $\zeta(\eta, t ; 0)=\zeta_{0}(\eta, t)$ to $\zeta(\eta, t ; 1)=\zeta(\eta, t)$ as $s$ approaches from 0 to 1 .

The auxiliary function $H(s)$ is chosen in the form:

$$
H(s)=\sum_{i=1}^{\infty} s^{i} C_{i}
$$

Here, $C_{1}, C_{2}, C_{3}, \ldots$ are constants. Next, we use the Taylor series to expand the function $\varphi(\eta, t ; s)$ about $s$ :

$$
\varphi(\eta, t ; s)=\zeta_{0}(\eta, t)+\sum_{j=1}^{\infty} \zeta_{j}\left(\eta, t ; C_{i}\right) s^{j}
$$

The nonlinear function $N(\zeta(\eta, t ; s))$ is decomposed as

$$
\begin{aligned}
N(\zeta(\eta, t ; s))= & N\left(\zeta_{0}(\eta, t)\right)+s\left[N\left(\zeta_{0}(\eta, t)+\zeta_{1}(\eta, t)\right)\right. \\
& \left.-N\left(\zeta_{0}(\eta, t)\right)\right]+s^{2}\left[N \left(\zeta_{0}(\eta, t)+\zeta_{1}(\eta, t)\right.\right. \\
& \left.\left.+\zeta_{2}(\eta, t)\right)-N\left(\zeta_{0}(\eta, t)+\zeta_{1}(\eta, t)\right)\right]+\cdots
\end{aligned}
$$

The expressions on the right-hand side of (6) are the DJ polynomials given as follows:

$$
\begin{array}{r}
N\left(\zeta_{0}(\eta, t)\right),\left[N\left(\zeta_{0}(\eta, t)+\zeta_{1}(\eta, t)\right)-N\left(\zeta_{0}(\eta, t)\right)\right], \\
{\left[N\left(\zeta_{0}(\eta, t)+\zeta_{1}(\eta, t)+\zeta_{2}(\eta, t)\right)-N\left(\zeta_{0}(\eta, t)+\zeta_{1}(\eta, t)\right)\right] .}
\end{array}
$$

In fact, the above polynomials are the terms of Taylor's series of the nonlinear term. The convergence of these polynomials was determined by Bhalekar and Daftardar-Gejji [24]. For simplification we expressed the polynomials as

$$
\begin{aligned}
& N_{0}=N\left(\zeta_{0}(\eta, t)\right), \\
& N_{m}=N\left(\sum_{i=0}^{m} \zeta_{i}(\eta, t)\right)-N\left(\sum_{i=0}^{m-1} \zeta_{i}(\eta, t)\right) .
\end{aligned}
$$

Now, we can also express

$$
N(\zeta(\eta, t ; s))=N_{0}+\sum_{k=1}^{\infty} s^{k} N_{k}
$$

Substituting equations (8)-(10) in equation (3) and comparing the like terms of $s$, we get the different order problems given as follows.

The zero-th order problem is

$$
\begin{aligned}
N\left(\zeta_{0}(\eta, t)\right)+g(\eta, t) & =0, \\
B\left(\zeta_{0}, \frac{\partial \zeta_{0}}{\partial t}\right) & =0 .
\end{aligned}
$$

The first-order problem is

$$
\begin{aligned}
& L\left(\zeta_{1}(\eta, t)\right)=C_{1} N_{0}\left(\zeta_{0}(\eta, t)\right), \\
& B\left(\zeta_{1}, \frac{\partial \zeta_{1}}{\partial t}\right)=0 .
\end{aligned}
$$

The second-order problem is

$$
\begin{aligned}
L\left(\zeta_{2}(\eta, t)\right)-L\left(\zeta_{1}(\eta, t)\right)= & C_{2} N_{0}\left(\zeta_{0}(\eta, t)\right)+C_{1}\left[L\left(\zeta_{1}(\eta, t)\right)\right. \\
& \left.+N_{1}\left(\zeta_{0}(\eta, t), \zeta_{1}(\eta, t)\right)\right], \\
B\left(\zeta_{2}, \frac{\partial \zeta_{2}}{\partial t}\right)= & 0 .
\end{aligned}
$$
using

The general governing equation for $\zeta_{j}(\eta, t)$ is given by

$$
\begin{aligned}
L\left(\zeta_{j}(\eta, t)\right)-L\left(\zeta_{j-1}(\eta, t)\right)= & C_{j} N_{0}\left(\zeta_{0}(\eta, t)\right) \\
& +\sum_{i=1}^{j-1} C_{i}\left[L\left(\zeta_{j-i}(\eta, t)\right)\right. \\
& +N_{j-i}\left(\zeta_{0}(\eta, t), \zeta_{1}(\eta, t),\right. \\
& \left.\left.\ldots, \zeta_{j-i}(\eta, t)\right)\right], \\
B\left(\zeta_{j}, \frac{\partial \zeta_{j}}{\partial t}\right)= & 0, \quad j=2,3, \ldots
\end{aligned}
$$


The solution of higher order problems can be easily estimated; however, the second-order solution gives encouraging results.

For $s=1$, equation (5) reduces to

$$
\tilde{\zeta}\left(\eta, t ; C_{i}\right)=\zeta_{0}(\eta, t)+\sum_{j \geq 1} \zeta_{j}\left(\eta, t ; C_{i}\right) .
$$

Replacing equation (15) into equation (2), we get the residual as

$$
\mathfrak{R}\left(\eta, t ; C_{i}\right)=L\left(\widetilde{\zeta}\left(\eta, t ; C_{i}\right)\right)+N\left(\widetilde{\zeta}\left(\eta, t ; C_{i}\right)\right)+g(\eta, t) .
$$

If $\Re\left(\eta, t ; C_{i}\right)=0$, then we get the exact solution. Different methods can be used to estimate the values of constants $C_{1}, C_{2}, C_{3}, \ldots, C_{j}$; but the method of least square is the most common method. In the method of least square, we minimize the errors by taking the square of the residuals over the given domain to get the following functional:

$$
\mathfrak{J}\left(C_{i}\right)=\int_{0}^{t} \int_{\Omega} \mathfrak{R}^{2}\left(\eta, t ; C_{i}\right) \mathrm{d} \eta \mathrm{d} t .
$$

Differentiating $J$ with respect to $C_{1}, C_{2}, C_{3}, \ldots, C_{j}$, we get the following system of equations containing $C_{1}, C_{2}, C_{3}, \ldots, C_{j}$ :

$$
\frac{\partial \mathfrak{I}}{\partial C_{1}}=\frac{\partial \mathfrak{I}}{\partial C_{2}}=\cdots=\frac{\partial \mathfrak{I}}{\partial C_{j}}=0 .
$$

Solving the above system, we get the values of $C_{1}, C_{2}, C_{3}, \ldots, C_{j}$. Replacing the values of $C_{1}, C_{2}, C_{3}, \ldots, C_{j}$ in equation (14), we get the approximate solution.

The method of least squares is a powerful technique and has been used in many other methods such as Optimal Homotopy Perturbation Method (OHPM) and Optimal Auxiliary Functions Method (OAFM) for calculating the optimum values of arbitrary constants $[25,26]$.

\section{Implementation of OHAM-DJ to a Coupled System of Nonlinear KdV Equations}

Problem 1. Consider system (1) with initial conditions [27]:

$$
\begin{aligned}
& \zeta(\eta, 0)=\frac{1}{3}\left(\omega-8 l^{2}\right)+4 l^{2} \tanh ^{2}(\eta \eta), \\
& \xi(\eta, 0)=\frac{-4 l^{2}\left(3 l^{2} C_{0}-2 \omega C_{2}+4 l^{2} C_{2}\right)}{3 C_{2}^{2}}+\frac{4 l^{2}}{C_{2}} \tanh ^{2}(\eta \eta), \\
& w(\eta, 0)=C_{0}+C_{2} \tanh ^{2}(\eta \eta) .
\end{aligned}
$$

Exact solution of equation (1) is

$$
\begin{aligned}
& \zeta(\eta, t)=\frac{1}{3}\left(\omega-8 l^{2}\right)+4 l^{2} \tanh ^{2}(l(\eta+\omega t)), \\
& \xi(\eta, t)=\frac{-4 l^{2}\left(3 l^{2} C_{0}-2 \omega C_{2}+4 l^{2} C_{2}\right)}{3 C_{2}^{2}}+\frac{4 l^{2}}{C_{2}} \tanh ^{2}((\eta+\omega t)), \\
& w(\eta, t)=C_{0}+C_{2} \tanh ^{2}(l(\eta+\omega t)),
\end{aligned}
$$

where $t=1, \omega=1.5, l=0.1, C_{0}=1.5$, and $C_{2}=0.1$ are different parameters. Applying the proposed method, we have the following.

The zero-th order problem is

$$
\begin{aligned}
\frac{\partial \zeta_{0}(\eta, t)}{\partial t} & =0, \\
\zeta_{0}(\eta, 0) & =\frac{1}{3}\left(\omega-8 l^{2}\right)+4 l^{2} \tanh ^{2}(l \eta), \\
\frac{\partial \xi_{0}(\eta, t)}{\partial t} & =0, \\
\xi_{0}(\eta, 0) & =\frac{-4 l^{2}\left(3 l^{2} C_{0}-2 \omega C_{2}+4 l^{2} C_{2}\right)}{3 C_{2}^{2}}+\frac{4 l^{2}}{C_{2}} \tanh ^{2}(l \eta), \\
\frac{\partial w_{0}(\eta, t)}{\partial t} & =0, \\
w_{0}(\eta, 0) & =C_{0}+C_{2} \tanh ^{2}(l \eta) .
\end{aligned}
$$

Its solution is

$$
\begin{aligned}
\zeta_{0}(\eta, t)= & 0.04000000000000001(11.83333333333333 \\
& \left.+1 \cdot \tanh ^{2}(0.1 \eta)\right), \\
\xi_{0}(\eta, t)= & 0.40000000000000001(0.83666666666666666 \\
& \left.+1 \cdot \tanh ^{2}(0.1 \eta)\right), \\
w_{0}(\eta, t)= & 0.1\left(15 .+1 \cdot \tanh ^{2}(0.1 \eta)\right) .
\end{aligned}
$$

The first-order problem is

$$
\begin{aligned}
& \frac{\partial \xi_{0}(\eta, t)}{\partial \eta}-3 C_{1} \xi_{0}(\eta, t) \frac{\partial w_{0}(\eta, t)}{\partial \eta}+0.5 C_{1} \frac{\partial^{3} \zeta_{0}(\eta, t)}{\partial \eta^{3}}=0, \zeta_{1}(\eta, 0)=0, \\
& -\frac{\partial \xi_{0}(\eta, t)}{\partial t}-C_{3} \frac{\partial \xi_{0}(\eta, t)}{\partial t}+\frac{\partial \xi_{1}(\eta, t)}{\partial t}+3 C_{3} \zeta_{0}(\eta, t) \frac{\partial \xi_{0}(\eta, t)}{\partial \eta} \\
& -C_{3} \frac{\partial^{3} \xi_{0}(\eta, t)}{\partial \eta^{3}}=0, \xi_{1}(\eta, 0)=0 . \\
& -\frac{\partial w_{0}(\eta, t)}{\partial \tau}-C_{5} \frac{\partial w_{0}(\eta, t)}{\partial t}+3 C_{5} \zeta_{0}(\eta, t) \frac{\partial w_{0}(\eta, t)}{\partial t}+\frac{\partial w_{1}(\eta, t)}{\partial t} \\
& -C_{5} \frac{\partial^{3} w_{0}(\eta, t)}{\partial \eta^{3}}=0, w_{1}(\eta, 0)=0 .
\end{aligned}
$$


TABLE 1: Absolute errors obtained by first-order OHAM-DJ and comparison is made with MOHAM of $\zeta(\eta, t)$ at $t=1, \omega=1.5, l=0.1, C_{0}=1.5$, and $C_{2}=0.1$.

\begin{tabular}{|c|c|c|c|c|}
\hline & \multicolumn{2}{|c|}{$t=0.1$} & \multicolumn{2}{|c|}{$t=0.01$} \\
\hline$\eta$ & MOHAM [27] & OHAM-DJ & MOHAM [27] & OHAM-DJ \\
\hline 0 & $8.8667 \times 10^{-4}$ & $8.99865 \times 10^{-6}$ & $8.99865 \times 10^{-6}$ & $8.99999 \times 10^{-8}$ \\
\hline 20 & $7.128 \times 10^{-4}$ & $1.1948 \times 10^{-4}$ & $8.06039 \times 10^{-5}$ & $1.20499 \times 10^{-5}$ \\
\hline 40 & $1.38951 \times 10^{-5}$ & $2.35856 \times 10^{-6}$ & $1.58421 \times 10^{-6}$ & $2.38 \times 10^{-7}$ \\
\hline 60 & $2.54789 \times 10^{-7}$ & $4.32582 \times 10^{-8}$ & $2.90535 \times 10^{-8}$ & $4.3652 \times 10^{-9}$ \\
\hline 80 & $4.66673 \times 10^{-9}$ & $7.92321 \times 10^{-10}$ & $5.32147 \times 10^{-10}$ & $7.99535 \times 10^{-11}$ \\
\hline 100 & $8.54742 \times 10^{-11}$ & $1.45118 \times 10^{-11}$ & $9.74665 \times 10^{-12}$ & $1.46427 \times 10^{-12}$ \\
\hline
\end{tabular}

TABle 2: Absolute errors obtained by first order OHAM-DJ and comparison is made with MOHAM of $\xi(\eta, t)$ at $t=1, \omega=1.5, l=0.1, C_{0}=1.5$, and $C_{2}=0.1$.

\begin{tabular}{|c|c|c|c|c|}
\hline \multirow[b]{2}{*}{$\eta$} & \multicolumn{2}{|c|}{$t=0.1$} & \multicolumn{2}{|c|}{$t=0.01$} \\
\hline & MOHAM [27] & OHAM-DJ & MOHAM [27] & OHAM-DJ \\
\hline 0 & $8.8667 \times 10^{-3}$ & $8.99865 \times 10^{-5}$ & $8.99865 \times 10^{-5}$ & $8.99999 \times 10^{-7}$ \\
\hline 20 & $7.128 \times 10^{-3}$ & $7.7428 \times 10^{-4}$ & $8.06039 \times 10^{-4}$ & $7.84417 \times 10^{-5}$ \\
\hline 40 & $1.38951 \times 10^{-4}$ & $1.50524 \times 10^{-5}$ & $1.58421 \times 10^{-5}$ & $1.52668 \times 10^{-6}$ \\
\hline 60 & $2.54789 \times 10^{-6}$ & $2.75994 \times 10^{-7}$ & $2.90535 \times 10^{-7}$ & $2.79932 \times 10^{-8}$ \\
\hline 80 & $4.66673 \times 10^{-8}$ & $5.05511 \times 10^{-9}$ & $5.32146 \times 10^{-9}$ & $5.12724 \times 10^{-10}$ \\
\hline 100 & $8.54741 \times 10^{-10}$ & $9.25876 \times 10^{-11}$ & $9.7466 \times 10^{-11}$ & $9.39082 \times 10^{-12}$ \\
\hline
\end{tabular}

TABLE 3: Absolute errors obtained by first order OHAM-DJ and comparison is made with MOHAM of $w(\eta, t)$ at $t=1, \omega=1.5, l=0.1, C_{0}=1.5$, and $C_{2}=0.1$.

\begin{tabular}{|c|c|c|c|c|}
\hline & \multicolumn{2}{|c|}{$t=0.1$} & \multicolumn{2}{|c|}{$t=0.01$} \\
\hline$\eta$ & MOHAM [27] & OHAM-DJ & MOHAM [27] & OHAM-DJ \\
\hline 0 & $2.2166 \times 10^{-3}$ & $2.2495 \times 10^{-5}$ & $2.2496 \times 10^{-5}$ & $2.5 \times 10^{-7}$ \\
\hline 20 & $1.782 \times 10^{-3}$ & $1.93585 \times 10^{-4}$ & $2.0151 \times 10^{-4}$ & $1.9611 \times 10^{-5}$ \\
\hline 40 & $3.4737 \times 10^{-5}$ & $3.76347 \times 10^{-6}$ & $3.9605 \times 10^{-6}$ & $3.8170 \times 10^{-7}$ \\
\hline 60 & $6.3697 \times 10^{-7}$ & $6.9005 \times 10^{-8}$ & $7.2633 \times 10^{-8}$ & $6.9989 \times 10^{-9}$ \\
\hline 80 & $1.1666 \times 10^{-8}$ & $1.2639 \times 10^{-9}$ & $1.3303 \times 10^{-9}$ & $1.2819 \times 10^{-10}$ \\
\hline 100 & $2.1368 \times 10^{-10}$ & $2.3149 \times 10^{-11}$ & $2.4366 \times 10^{-11}$ & $23479 \times 10^{-12}$ \\
\hline
\end{tabular}

Solution of the first-order problem is given as

$$
\begin{aligned}
\zeta_{1}\left(\eta, t, C_{1}\right)= & 0.00032000000000000013 t\left(473.2499999999999 C_{1} \operatorname{sech}^{2}(0.1 \eta) \tanh (0.1 \eta)+1 . C_{1} \operatorname{sech}^{4}(0.1 \eta) \tanh (0.1 \eta)\right. \\
& \left.+102.49999999999999 C_{1} \operatorname{sech}^{2}(0.1 \eta) \tanh ^{3}(0.1 \eta)\right) \\
\xi_{1}\left(\eta, t, C_{3}\right)= & -0.006400000000000002 t\left(1 . C_{3} \operatorname{sech}^{4}(0.1 \eta) \tanh (0.1 \eta)-0.5 C_{3} \operatorname{sech}^{2}(0.1 \eta) \tanh (0.1 \eta)\right), \\
w_{1}\left(\eta, t, C_{5}\right)= & -0.0016000000000000005 t\left(1 . C_{5} \operatorname{sech}^{4}(0.1 \eta) \tanh (0.1 \eta)-0.5 C_{3} \operatorname{sech}^{2}(0.1 \eta) \tanh ^{3}(0.1 \eta)\right) .
\end{aligned}
$$

Adding equations (22) and (24), we get first-order approximate solution by OHAM-DJ as

$$
\begin{aligned}
\tilde{\zeta}(\eta, t) & =\zeta_{0}(\eta, t)+\zeta_{1}\left(\eta, t, C_{1}\right), \\
\tilde{\xi}(\eta, t) & =\xi_{0}(\eta, t)+\xi_{1}\left(\eta, t, C_{3}\right), \\
\widetilde{w}(\eta, t) & =w_{0}(\eta, t)+w_{1}\left(\eta, t, C_{5}\right) .
\end{aligned}
$$

The values of convergence control constants are calculated using the method of least squares whose values are given as follows:

$$
\begin{aligned}
& C_{1}=-0.03137136500930365, \\
& C_{3}=1.8490804836636396, \\
& C_{5}=1.8456318876033717 .
\end{aligned}
$$

\section{Results and Discussion}

OHAM-DJ has been extended for the solution of coupled system of $\mathrm{KdV}$ equations. Tables 1-3 show the comparison of absolute errors of first-order approximate solutions 


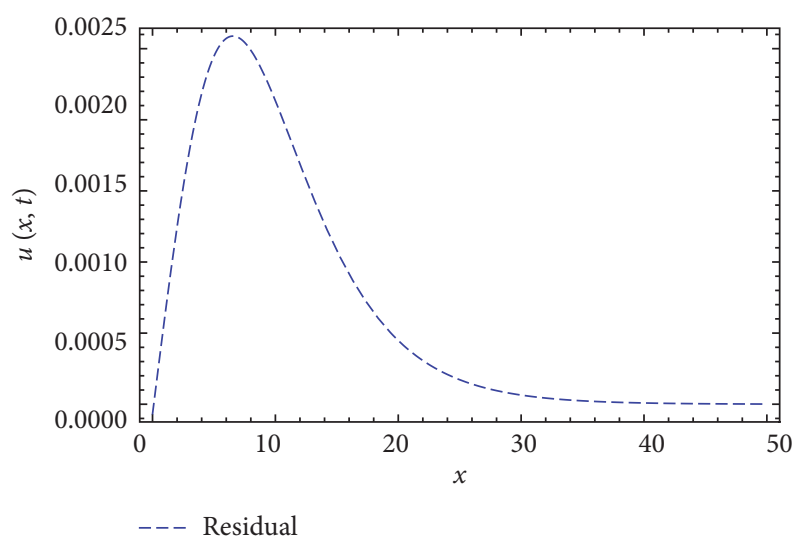

Figure 1: Residual of $\zeta(\eta, t)$ by OHAM-DJ at $t=0.1$.

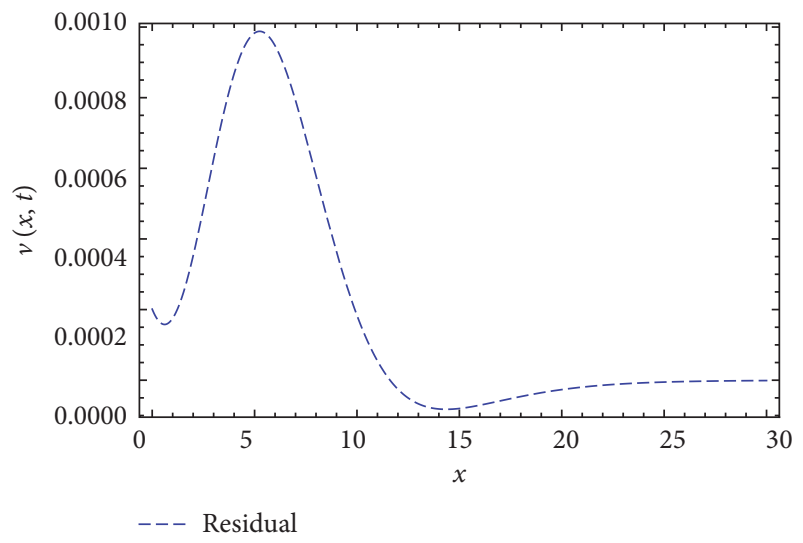

Figure 2: Residual of $\xi(\eta, t)$ by OHAM-DJ at $t=0.1$.

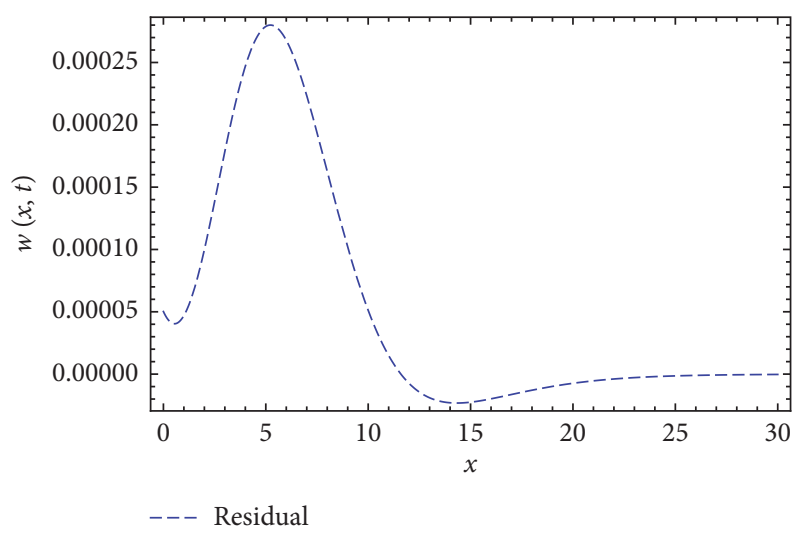

Figure 3: Residual of $w(\eta, t)$ by OHAM-DJ at $t=0.1$.

$\zeta(\eta, t), \xi(\eta, t)$, and $w(\eta, t)$ by OHAM-DJ with MOHAM. Figures 1-3 show the residual graphs for $\zeta(\eta, t)$, $\xi(\eta, t)$, and $w(\eta, t)$ at $t=0.1$. It is worth pointing out that OHAM-DJ grants fast convergence solutions than OHAM.

\section{Conclusions}

OHAM-DJ has been successfully applied for the solution of a coupled nonlinear $\mathrm{KdV}$ system. The proposed method contains an adaptable auxiliary function that is used to control the convergence of the solution and grants alteration inside the convergence region wherever it is required. This strategy is free from small parameter assumption and does not need any initial guess. The proposed method does not use discretization, and the convergence is controlled by selfassertive constants. Results revealed that OHAM-DJ is very consistent in comparison with MOHAM. The proposed method is an essential analytical method and well-organized in finding the solutions for an extensive class of coupled systems of PDEs. The accuracy of the proposed method can be further improved by taking higher order approximations. Extension of OHAM-DJ to the coupled nonlinear Kortewegde-Vries system is more accurate and as a result, it will be more appealing for researchers to apply this method to the coupled system of partial differential equations arising in different fields of engineering sciences.

\section{Data Availability}

All the data and meta-data concerning the findings of the manuscript are given in the manuscript.

\section{Conflicts of Interest}

The authors declared that they have no conflicts of interest.

\section{Authors' Contributions}

Zawar Hussain carried out the simulations. Rashid Nawaz linguistically edited and sequenced the paper. Abraiz Khattak made the corrections in the paper, and Adam Khan drafted the paper.

\section{References}

[1] J. C. Butcher, The Numerical Analysis of Ordinary Differential Equations: Rung-Kutta and General Linear Methods, WileyInterscience, Hoboken, NJ, USA, 1987.

[2] E. Hairer, M. Roche, and C. Lubich, The Numerical Solution of Differential-Algebraic Systems by Runge-Kutta Methods, Springer, Berlin, Germany, 1989.

[3] S. N. Ha, "A nonlinear shooting method for two-point boundary value problems," Computers \& Mathematics with Applications, vol. 42, no. 10-11, pp. 1411-1420, 2001.

[4] M. R. Osborne, "On shooting methods for boundary value problems," Journal of Mathematical Analysis and Applications, vol. 27, no. 2, pp. 417-433, 1969.

[5] V. Thomée, "From finite differences to finite elements a short history of numerical analysis of partial differential equations," Journal of Computational and Applied Mathematics, vol. 128, no. 1-2, pp. 1-54, 2001.

[6] Q. Fang, T. Tsuchiya, and T. Yamamoto, "Finite difference, finite element and finite volume methods applied to two-point boundary value problems," Journal of Computational and Applied Mathematics, vol. 139, no. 1, pp. 9-19, 2002.

[7] M. Dehghan, "Weighted finite difference techniques for the one-dimensional advection-diffusion equation," Applied Mathematics and Computation, vol. 147, no. 2, pp. 307-319, 2004. 
[8] F. Ihlenburg and I. Babuška, "Finite element solution of the Helmholtz equation with high wave number," Computers \& Mathematics with Applications, vol. 30, no. 9, pp. 9-37, 1995.

[9] Y. W. Hwon and H. Bank, The Finite Element Method Using MATLAB, CRC Press, New York, NY, USA, 1996.

[10] H.-Y. Hu and Z.-C. Li, "Collocation methods for Poisson's equation," Computer Methods in Applied Mechanics and Engineering, vol. 195, no. 33-36, pp. 4139-4160, 2006.

[11] M. Uddin, S. Haq, and S. Siraj-ul-Islam, "Numerical solution of complex modified Korteweg-de Vries equation by meshfree collocation method," Computers \& Mathematics with Applications, vol. 58, no. 3, pp. 566-578, 2009.

[12] R. Alt and J. Vignes, "Validation of results of collocation methods for ODEs with the CADNA library," Applied Numerical Mathematics, vol. 21, no. 2, pp. 119-139, 1996.

[13] J. Goh, A. A. Majid, and A. I. Ismail, "Cubic B-spline collocation method for one-dimensional heat and advectiondiffusion equations," Journal of Applied Mathematics, vol. 2012, Article ID 4587018, 8 pages, 2012.

[14] S. J. Liao, The proposed homotopy analysis technique for the solution of nonlinear problems, Ph.D. thesis, Shanghai Jiao Tong University, Shanghai, China, 1992.

[15] S. J. Liao, "A kind of linearity-invariance under homotopy and some simple applications of it in mechanics," Technical Report 520, Institute of Ship Building, University of Hamburg, Hamburg, Germany, 1992.

[16] V. Marinca and N. Herişanu, "Application of optimal homotopy asymptotic method for solving nonlinear equations arising in heat transfer," International Communications in Heat and Mass Transfer, vol. 35, no. 6, pp. 710-715, 2008.

[17] V. Marinca, N. Herişanu, C. Bota, and B. Marinca, "An optimal homotopy asymptotic method applied to steady flow of a fourth-grade fluid past a porous plate," Applied Mathematics Letters, vol. 22, no. 2, pp. 245-251, 2009.

[18] N. Herisanu, V. Marinca, T. Dordea, and G. Madescu, "A new analytical approach to nonlinear vibration of an electric machine," Romanian Academy Series A-Mathematics Physics Technical Sciences Information Science, vol. 9, pp. 229-236, 2008.

[19] V. Marinca and N. Herişanu, "On the flow of a Walters-type B' viscoelastic fluid in a vertical channel with porous wall," International Journal of Heat and Mass Transfer, vol. 79, pp. 146-165, 2014.

[20] N. Herisanu, V. Marinca, and G. Madescu, "Nonlinear dynamics of a wind turbine permanent magnet generator system in different wind Profile conditions," AIP Conference Proceedings, vol. 1863, 2017.

[21] J. Ali, S. Shah, S. Islam, and H. Khan, "Application of optimal homotopy asymptotic method with daftardar-jeffery polynomials to non-linear differential equations," World Applied Sciences Journal, vol. 28, pp. 1456-1462, 2013.

[22] M. Abdou and A. Elgarayhi, "On optimal homotopy asymptotic method of quantum Zakharov-Kuznetsov equation in ion acoustic waves," Walailak Journal of Science and Technology, vol. 13, no. 9, 2015.

[23] Z. Shah, R. Nawaz, S. Shah, S. I. A. Shah, and M. Shah, "Use of the daftardar-jeffery polynomials in optimal homotopy asymptotic method for the solution of linear and nonlinear klein-gordon equations," Journal of Applied Environmental and Biological Sciences, vol. 6, pp. 71-81, 2016.

[24] S. Bhalekar and V. Daftardar-Gejji, "Convergence of the new iterative method," International Journal of Differential Equations, vol. 2011, Article ID 989065, 10 pages, 2011.
[25] F. Ghani, C. L. Ali, M. M. Rashidi, and T. Hajjari, “Application of modified optimal homotopy perturbation method to higher order boundary value problems in a finite domain," Hacettepe Journal of Mathematics and Statistics, vol. 5, no. 45, pp. 1049-1060, 2016.

[26] N. Herisanu, V. Marinca, G. Madescu, and F. Dragan, "Dynamic response of a permanent magnet synchronous generator to a wind gust," Energies, vol. 12, no. 5, p. 915, 2019.

[27] M. Fizza, H. Ullah, S. Islam, Q. Shah, F. I. Chohan, and M. B. Mamat, "Modifications of the multistep optimal homotopy asymptotic method to some nonlinear $\mathrm{KdV}$ equations," Journal of Pure and Applied Mathematics, vol. 11, no. 2, pp. 537-552, 2018. 